\title{
Lattice Solitons in Quasicondensates
}

\author{
V. Ahufinger and A. Sanpera \\ Institut für Theoretische Physik, Universität Hannover, D-30167 Hannover, Germany
}

(Received 12 October 2004; published 8 April 2005)

\begin{abstract}
We analyze finite temperature effects in the generation of bright solitons in condensates in optical lattices. We show that even in the presence of strong phase fluctuations solitonic structures with a well defined phase profile can be created. We propose a novel family of variational functions which describe well the properties of these solitons and account for the nonlinear effects in the band structure. We discuss also the mobility and collisions of these localized wave packets.
\end{abstract}

DOI: 10.1103/PhysRevLett.94.130403

Bose-Einstein condensates (BEC) in optical lattices (OL) are unique candidates to explore phenomena that are often extremely elusive in other areas of physics. The dynamics of the system is dominated by the interplay between the nonlinearity that can be modified via Feshbach resonances [1] and the periodicity which can be engineered through the intensity, geometry, polarization, and phase of the two counterpropagating laser beams conforming the optical lattice. BEC in optical lattices exhibit phenomena known from solid state physics as demonstrated, for instance, in [2]. Furthermore, its similarity to other cubic nonlinear periodic media has stimulated a renewed interest in solitonic models [3-7].

Solitons, which, strictly speaking, are exact solutions of integrable models corresponding to wave packets that propagate without change of their shapes and velocities even in the presence of collisions, appear in many branches of physics. In one-dimensional homogeneous condensates with attractive interactions, bright solitons exist [8] and have been recently observed [9]. The presence of a periodic potential, like, e.g., an optical lattice, breaks the translational invariance, and the system becomes most likely nonintegrable, having less conserved quantities than degrees of freedom. Nonintegrable systems, however, admit also localized solutions which are as well commonly termed solitons. These structures differ from proper solitons either in their motion and/or their collisions. A well known example are optical solitons in periodic media (cf. [10]) whose interactions have been extensively studied using either the discrete nonlinear Schrödinger (DNLS) equation [11] or weakly perturbed integrable models [12].

So far, to our knowledge, the generation of lattice solitons in condensates has been only discussed at zero temperature [3-7], where there is an analogy between an array of optical waveguides in a Kerr medium and a BEC in a periodic potential. In this limit, one can generate [13] bright lattice solitons in repulsive condensates when the tunneling rate balances the nonlinear energy of the system. This compensation occurs if the soliton is placed at the edge of the first Brillouin zone where the effective mass becomes negative reaching the staggered configuration [14].
PACS numbers: 03.75.Lm, 03.75.Kk, 05.30.Jp, 05.45.Yv

Here we discuss the generation of solitons in repulsive condensates at finite temperature. We show that even when the condensate is not phase coherent, solitonic structures with well defined phase profiles can be created. A new insight into the nature of these lattice solitons is obtained by means of a novel variational ansatz that accounts for the effects of the nonlinearity. Finally, we address the issue of mobility and collisions.

For BEC in 3D trapping geometries, fluctuations of density and phase are only important in a narrow temperature range near the BEC transition temperature $T_{c}$ [15]. For pure 1D systems, however, phase fluctuations are present at temperatures far below the degeneracy temperature $T_{d}=$ $N \hbar \omega_{x} / k_{B}$, while density fluctuations are still suppressed [16] ( $k_{B}$ denotes the Boltzmann constant, $N$ the number of atoms, $\omega_{x}$ the axial trapping frequency). Phase fluctuations can be studied by solving the Bogoliubov-de Gennes $(\mathrm{BdG})$ equations describing elementary excitations. Writing the quantum field operator as $\hat{\varphi}(x)=$ $\sqrt{n_{0}(x)} \exp [i \hat{\phi}(x)]$, where $n_{0}(x)$ denotes the density, the phase operator takes the form [17]:

$$
\hat{\phi}(x)=\frac{1}{\sqrt{4 n_{0}(x)}} \sum_{j=1}^{\infty} f_{j}^{+}(x) \hat{a}_{j}+\text { H.c. }
$$

where $\hat{a}_{j}$ is the annihilation operator of the excitation with quantum number $j$ and energy $\epsilon_{j}=\hbar \omega_{x} \sqrt{j(j+1) / 2}$, and $f_{j}^{+}=u_{j}+v_{j}$, where $u_{j}$ and $v_{j}$ denote the excitation functions determined by the BdG equations. In $1 \mathrm{D}$ and in the Thomas-Fermi (TF) regime, the functions $f_{j}^{+}$have the form:

$$
f_{j}^{+}(x)=\sqrt{\frac{(j+1 / 2) 2 \mu}{R_{\mathrm{TF}} \epsilon_{j}}\left[1-\left(\frac{x}{R_{\mathrm{TF}}}\right)^{2}\right]} P_{j}\left(\frac{x}{R_{\mathrm{TF}}}\right),
$$

where $P_{j}\left(x / R_{\mathrm{TF}}\right)$ are Legendre polynomials, $R_{\mathrm{TF}}=$ $\left(2 \mu / m \omega_{x}^{2}\right)^{1 / 2}$ is the TF radius, and $\mu$ the chemical potential. The phase coherence length, $L_{\phi}=R_{\mathrm{TF}} T_{d} \hbar \omega_{x} / \mu T$, characterizes the maximal distance between two phase correlated points in the condensate. Phase fluctuations increase for large trap aspect ratios, $\omega_{t} / \omega_{x}$, and small $N$ [18]. 
To study temperature effects in the generation of lattice solitons, we consider a ${ }^{87} \mathrm{Rb}$ condensate with $N=500$ atoms in a magnetic trap with frequencies $\omega_{t}=$ $715 \times 2 \pi \mathrm{Hz}$ and $\omega_{x}=14 \times 2 \pi \mathrm{Hz}$. For such parameters, the system is effectively in a $1 \mathrm{D}$ regime since transverse excitations are suppressed $\left(\mu \ll \hbar \omega_{t}\right)$. Moreover, along the axial direction, the condensate is well in the TF regime $\left(\mu \gg \hbar \omega_{x}\right)$. To create lattice solitons in repulsive condensates, the optical lattice depth belongs to the socalled weak potential regime. Thus, one cannot use the tight binding approximation [19] to rewrite the condensate order parameter as a sum of wave functions localized in each well of the lattice. To study the dynamics of the system we use the 1D Gross-Pitaevskii equation (GPE):

$$
i \hbar \dot{\varphi}(x, t)=\left[-\frac{\hbar^{2}}{2 m} \nabla+V(x, t)+g|\varphi(x, t)|^{2}\right] \varphi(x, t),
$$

with coupling constant $g=2 N \hbar a_{s} \omega_{t}$ and scattering length $a_{s}=5.8 \mathrm{~nm}$. The confining potential $V(x, t)=$ $m \omega_{x}(t)^{2} x^{2} / 2+V_{0}(t) \sin ^{2}(\pi x / d)$ describes both the axial magnetic trap and the optical lattice. The later is characterized by its maximal depth $V_{0}=1 E_{r}$ and by its spatial period $d=\lambda / 2(\lambda=795 \mathrm{~nm})$. Energy is expressed in units of the recoil energy $E_{r}=\hbar^{2} k^{2} / 2 m$, where $k=$ $\pi / d$. Temperature is included at the level of the GPE by calculating first the density at $T=0$ in the presence of the magnetic trap only, i.e., $V(x, t=0)=m \omega_{x}^{2} x^{2} / 2$ and then the phase operator (1). To this aim, we calculate the Bose occupation $N_{j}=\left[e^{\epsilon_{j} / k_{B}}-1\right]^{-1}$ of the low-energy modes in the Bogoliubov approximation for a fixed $T$ replacing the operators $\hat{a}_{j}$ and $\hat{a}_{j}^{+}$by random complex variables $\alpha_{j}$ and $\alpha_{j}^{*}$ such that $\left\langle\left|\alpha_{j}\right|^{2}\right\rangle=N_{j}[18]$. One can also study the quasi 1D case using a 3D GPE and replacing in (1) the energy of low excitations by $\epsilon_{j}=\hbar \omega_{x} \sqrt{j(j+3) / 4}$ [20] and the Legendre by Jacobi polynomials [18].

We summarize the procedure to generate lattice solitons at a given $T$. Once the phase fluctuations have been included, periodicity is introduced by growing adiabatically the optical lattice $(t \leq \tau)$. During this process, density fluctuations arise. We simultaneously turn off the magnetic trap and imprint a phase difference of $\pi$ between consecutive wells to place the condensate at the edge of the first Brillouin zone. This is achieved by using an auxiliary optical lattice with double spatial period than the first one, acting for a time much shorter than the tunneling and the correlation time of the system [4]. Hence, the condensate acquires the desired phase without modifying its density profile.

Figure 1 shows density and phase of the condensate at $T=0.8 T_{c}\left(T_{c}=N \hbar \omega_{x} / k_{B} \ln 2 N\right.$ [21]) (a) in the presence of the magnetic trap only $(t=0)$, (b) after growing adiabatically the optical lattice $(t=\tau)$, and (c) $100 \mathrm{~ms}$ after the phase imprint has been performed and the magnetic trap has been switched off $(t=\tau+100)$. Figure 1(d) shows a lattice soliton generated at $T=0$ with otherwise identical parameters. Despite the strong fluctuations of phase and density induced by finite temperature, the system (after phase imprint) evolves towards a staggered soliton configuration. It contains approximately $35 \%$ of the initial atoms and remains so for times much larger than the tunneling time. This robustness can be understood by realizing that the size of the lattice soliton is smaller than the phase coherence length for all $T<T_{c}$. The soliton size (which depends on $N$, being smaller as $N$ increases) is independent of the temperature but due to the random character of the fluctuations, the position of the generated soliton is different for each realization.

An analytical description of lattice solitons in repulsive condensates is often performed through an effective theory for the soliton's envelope in the effective mass approximation [22]. The influence of the periodic potential is included there via an effective mass and coupling constant.
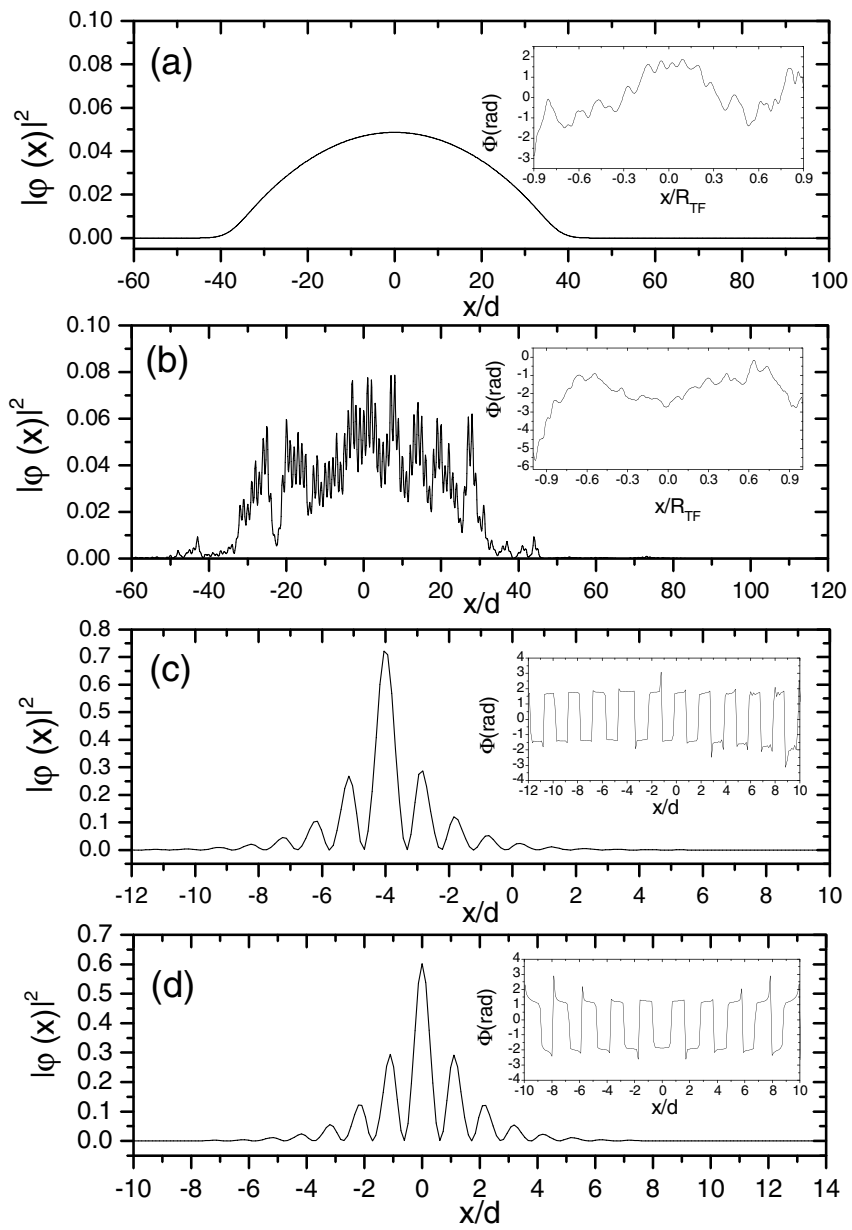

FIG. 1. Density and phase (inset) profile (a) of magnetically trapped $\left(\omega_{x}=14 \times 2 \pi \mathrm{Hz}\right){ }^{87} \mathrm{Rb}$ ground state condensate with $N=500$ at $T=0.8 T_{c}$, (b) after the adiabatic growing of the optical lattice $\left(V_{0}=1 E_{r}\right.$ and $\left.\lambda=795 \mathrm{~nm}\right)$, (c) of lattice soliton $100 \mathrm{~ms}$ after the imprinting of a phase difference of $\pi$ between consecutive wells and after the magnetic trap is switched off, and (d) of lattice soliton generated under the same conditions of (c) but at $T=0$. 
Instead let us consider a very general ansatz for the soliton wave function:

$$
\varphi(x, t)=e^{-i \mu t / \hbar} G\left(A, \sigma, x, x_{0}\right) F(x),
$$

where $G\left(A, \sigma, x, x_{0}\right)$ describes the envelope of a soliton of amplitude $A$, width $\sigma$, and centered at $x_{0}$. The effect of the periodic potential is included, in the weak approximation limit, as a combination of linear Bloch functions $F(x)=$ $\sum_{k} e^{i k x} f_{k}(x)$, where the $f_{k}(x)$ have the periodicity of the lattice. At the edge of the first Brillouin zone, this combination can be approximated by only two harmonics, i.e., $F(x)=\cos (2 \pi x / \lambda)$ [5]. Notice that such an ansatz does not take into account nonlinear effects. Moreover, it does not present a minimum in the energy functional of the GPE for any atom number. Inspection of Fig. 1(c) and 1(d) shows that the density profile inside each well is shifted with respect to the minimum of the optical potential. A relative simple function that reproduces such site dependent shift is $F(x)=\cos \left[2 \pi x / \lambda^{\prime}(x)\right]$ with an effective wavelength $\lambda^{\prime}(x)=\lambda\left[1+\alpha(N)\left|G\left(A, \sigma, x, x_{0}\right)\right|^{2}\right]$, where $\alpha(N) \ll 1$ is a variational parameter which depends only on $N$. In other words, the modification of the band structure due to the nonlinearity results in an effective change of the periodicity of the system. The ansatz (4) with the effective wavelength $\lambda^{\prime}(x)$ does not allow a fully analytical treatment for the energy functional. As a first approximation we assume thus that the effect of the nonlinearity is to shift $\lambda$ simply by a constant $\lambda^{\prime}=\lambda+\delta$. With this new ansatz, imposing the normalization of the wave function (i.e., conservation of $N$ ) and assuming a Gaussian envelope $G\left(A, \sigma, x, x_{0}\right)=A \exp \left[\left(x-x_{0}\right)^{2} / 2 \sigma^{2}\right]$, the energy functional $E\left[A, \sigma, x_{0}, \delta\right]$ reads:

$$
\begin{aligned}
E= & \int\left[\frac{\hbar^{2}}{2 m}|\nabla \varphi(x)|^{2}+\frac{g}{2}|\varphi(x)|^{4}+V(x)|\varphi(x)|^{2}\right] d x \\
= & B\left(\frac{\hbar^{2}}{m}\left[\frac{1+e^{-k^{\prime 2} \sigma^{2}} \cos \left(2 k^{\prime} x_{0}\right)}{2 \sigma^{2}}+k^{\prime 2}\right]\right. \\
& +\frac{g|A|^{2}}{4 \sqrt{2}}\left[3+e^{-2 k^{\prime 2} \sigma^{2}} \cos \left(4 k^{\prime} x_{0}\right)+4 e^{-\frac{k^{\prime 2} \sigma^{2}}{2}} \cos \left(2 k^{\prime} x_{0}\right)\right] \\
& +V_{0}\left[1+e^{-k^{\prime 2} \sigma^{2}} \cos \left(2 k^{\prime} x_{0}\right)-e^{-k^{2} \sigma^{2}} \cos \left(2 k x_{0}\right)\right. \\
& \left.\left.-\frac{1}{2} e^{-k_{-}^{2} \sigma^{2}} \cos \left(2 k_{-} x_{0}\right)-\frac{1}{2} e^{-k_{+}^{2} \sigma^{2}} \cos \left(2 k_{+} x_{0}\right)\right]\right),
\end{aligned}
$$

where $B=|A|^{2} \sqrt{\pi} \sigma / 4, k^{\prime}=2 \pi / \lambda^{\prime}$, and $k_{ \pm}=k^{\prime} \pm k$. We minimize (5) for a soliton centered in one lattice site, i.e., $x_{0}=0$, as a function of the shift $\delta$ and the width $\sigma$. The other free parameter $A$, the amplitude, is fixed by normalization, i.e., $1 \equiv 2 B\left[1+e^{-k^{\prime 2} \sigma^{2}} \cos \left(2 k^{\prime} x_{0}\right)\right]$.

For any $N$ and $x_{0}$, minimization of $E\left[A, \sigma, x_{0}, \delta\right]$ with respect to $\sigma$ and $\delta$ presents now a clear minimum. The values of $\sigma$ and $\delta$ obtained through minimization coincide with the values of the width and on-site shift obtained by solving numerically the GPE (3). Also, minimization of (5) correctly indicates that solitons containing few atoms $(N<$
$100)$ are very extended (large $\sigma$ ) and present practically no shift ( $\delta \leq 0.001$ ), as one should expect in the continuum limit. On the contrary for larger $N, \sigma$ decreases and $\delta$ grows.

The Peierls-Nabarro (PN) barrier [4,23], i.e., the energy difference between a solitonic configuration centered in one minimum of the lattice, $E\left[A, \sigma, x_{0}=n d, \delta\right]$, and the one centered in one maximum, $E\left[A^{\prime}, \sigma^{\prime}, x_{0}=n d / 2, \delta^{\prime}\right]$, has been also calculated using (5). For any value of $N$, $E\left[A, \sigma, x_{0}=n d, \delta\right]<E\left[A^{\prime}, \sigma^{\prime}, x_{0}=n d / 2, \delta^{\prime}\right]$. Thus, even the constant shift approximation reproduces the main features of lattice solitons.

On-site lattice solitons are created initially at rest. Nevertheless, if they are well localized in momentum space and an instantaneous transfer of momentum (large enough to overcome the PN barrier) is given to the system, the solitons move in opposite direction to the given momentum due to their negative effective mass. Notice that the variational ansatz $F(x)=\cos \left(2 \pi x / \lambda^{\prime}\right)$ with $\lambda^{\prime}=\lambda+\delta$ is meaningful only in the static case. Such a simplified solution cannot be used to study soliton dynamics, since the soliton is always chirped with respect to its center. The Euler-Lagrange equations for an ansatz whose periodicity depends also on the density are quite complex. Therefore, to study dynamical behavior, one has to rely on numerical simulations.

Giving momentum to the system is generally accompanied by radiation of atoms. The larger the given momentum is, the larger the losses. For small initial transfer of momentum the system exhibits a nonlinear response, its velocity slows down, and eventually a complete halt of the soliton occurs. The given momentum $p$, for which the soliton experiences a nonlinear response lies on the range $0 \leq p \leq \hbar\left(k-k^{\prime}\right)$, where $k^{\prime}$ corresponds to the inverse of the effective wavelength. Thus the linear response is recovered for broad solitons since $k \simeq k^{\prime}$ and for well localized solitons if the initial given momentum is large enough, i.e., $p>\hbar\left(k-k^{\prime}\right)$. Recently nonlinear movement of discrete solitons has also been reported [5]. The interaction between lattice solitons depends also strongly on the initial given momentum. We study numerically collisions between two identical solitons with $N=200$ each, created initially at rest and separated by a distance $2 x_{0}=246 \mathrm{~d}$. For small values of the initial momentum $p \simeq 0.1 k \hbar$, the two initial solitons merge at the interaction point resulting in a single soliton (with $N=200$ ) at rest. The excess of atoms is lost by radiation in a symmetric way [Fig. 2(a)]. For intermediate values of the initial kick, $p \simeq 0.2 k \hbar$, the two solitons merge in a moving soliton with smaller $N$ than the initial ones. For larger kicks, $p \simeq 0.3 k \hbar$, the solitons exhibit a quasielastic collision where the two solitons pass each other. The average phase difference between the two solitons also affects the nature of their interactions. For $p=0.1 k \hbar$ and identical initial phase distribution, one obtains the fusion of the initial two solitons into a single one at rest [Fig. 2(a)]. This merging behavior is always 

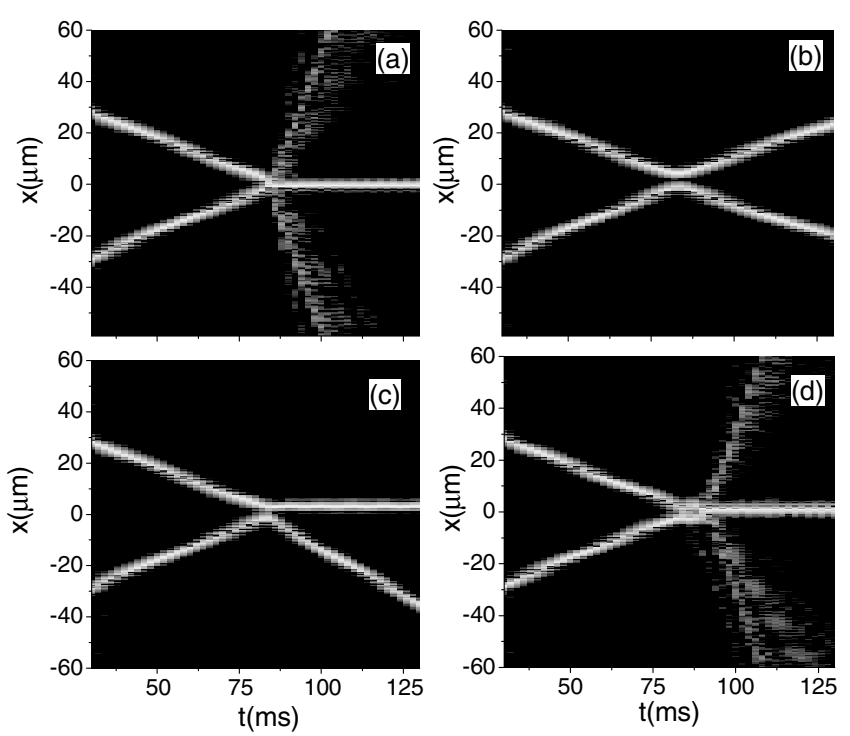

FIG. 2. Collisions between two identical lattice solitons $(N=$ 200) at an initial distance of $2 x_{0}=246 d$ and with an initial phase difference of (a) $\Delta \phi=0 \mathrm{rad}$, (b) $\Delta \phi=\pi / 2 \mathrm{rad}$, (c) $\Delta \phi=0.3 \mathrm{rad}$, and (d) $\Delta \phi=0.9 \mathrm{rad}$. The initial transfer of momentum is $0.1 \mathrm{k \hbar}$ in all the cases.

present for average phase difference between the solitons below $0.19 \mathrm{rad}$, although the final structure can either be a soliton or a breather and its position can also change. For phase differences of the order of $\pi / 2$ or larger, the two solitons always repel each other [Fig. 2(b)]. Between these two extreme cases, the dynamics of the system is unpredictable. Two solitons either with approximately the same $N$ and same velocity or with a different $N$ (being the one with more atoms the slower) [Fig. 2(c)] are examples of possible situations after the collision. In other cases, the two initial solitons form a bound state that ends up into a single soliton [Fig. 2(d)]. These results evidence the strong dependence on the initial conditions in the dynamics of the system.

Summarizing, finite temperature effects in the generation of lattice solitons in quasicondensates have been addressed. New insight into the nature of these structures is provided through a new family of variational functions in which the effect of the nonlinearity is shown as an effective change in the lattice periodicity. We hope that such a novel ansatz opens new possibilities in the study of lattice solitons.

We thank M.K. Oberthaler, E. Ostrovskaya, and M. Lewenstein for stimulating discussions. We acknowledge support from Deutsche Forschungsgemeinschaft (SFB 407) and from the ESF Programme QUDEDIS. V. A. acknowledges support from the European Community (MEIF-CT-2003 -501075).
[1] S. Inouye et al., Nature (London) 392, 151 (1998); S. L. Cornish et al., Phys. Rev. Lett. 85, 1795 (2000).

[2] B. P. Anderson and M. A. Kasevich, Science 282, 1686 (1998); O. Morsch et al., Phys. Rev. Lett. 87, 140402 (2001); F. S. Cataliotti et al., Science 293, 843 (2001).

[3] O. Zobay et al., Phys. Rev. A 59, 643 (1999); A. Trombettoni and A. Smerzi, Phys. Rev. Lett. 86, 2353 (2001); F. Kh. Abdullaev et al., Phys. Rev. A 64, 043606 (2001); E. A. Ostrovskaya and Y.S. Kivshar, Phys. Rev. Lett. 90, 160407 (2003).

[4] V. Ahufinger et al., Phys. Rev. A 69, 053604 (2004).

[5] M. J. Ablowitz, Z. H. Musslimani, and G. Biondini, Phys. Rev. E 65, 026602 (2002); H. Sakaguchi and B.A. Malomed, J. Phys. B 37, 1443 (2004).

[6] B. J. Dabrowska, E. A. Ostrovskaya, and Y.S. Kivshar, cond-mat/0408234.

[7] B. B. Baizakov, V. V. Konotop, and M. Salerno, J. Phys. B 35, 5105 (2002); B. B. Baizakov, B. A. Malomed, and M. Salerno, Europhys. Lett. 63, 642 (2003); H. Sakaguchi and B. A. Malomed, J. Phys. B 37, 2225 (2004); A. M. Dudarev, R. B. Diener, and Q. Niu, J. Opt. B 6, S231 (2004).

[8] V. E. Zakharov and A. B. Shabat, Sov. Phys. JETP 37, 823 (1973).

[9] L. Khaykovich et al., Science 296, 1290 (2002); K. E. Strecker et al., Nature (London) 417, 150 (2002).

[10] Y.S. Kivshar and G. Agrawal, Optical Solitons (Academic, New York, 2003).

[11] A. B. Aceves et al., Phys. Rev. E 53, 1172 (1996); D. Cai, A. R. Bishop, and N. Grønbech-Jensen, Phys. Rev. E 56, 7246 (1997); M. J. Ablowitz and Z. H. Musslimani, Phys. Rev. E 65, 056618 (2002); I. E. Papacharalampous et al., Phys. Rev. E 68, 046604 (2003).

[12] D. A. Segamin et al., Physica B (Amsterdam) 316, 136 (2002); S. V. Dmitriev et al., Phys. Rev. E 66, 046609 (2002); S. V. Dmitriev and T. Shigenari, Chaos 12, 324 (2002); S. V. Dmitriev et al., nlin.PS/0309054.

[13] B. Eiermann et al., Phys. Rev. Lett. 92, 230401 (2004).

[14] Y. S. Kivshar, Opt. Lett. 18, 1147 (1993); J. W. Fleischer et al., Phys. Rev. Lett. 90, 023902 (2003).

[15] D. S. Petrov, G. V. Shlyapnikov, and J. T. M. Walraven, Phys. Rev. Lett. 87, 050404 (2001).

[16] D. S. Petrov, G. V. Shlyapnikov, and J. T. M. Walraven, Phys. Rev. Lett. 85, 3745 (2000).

[17] S. I. Shevchenko, Sov. J. Low Temp. Phys. 18, 223 (1992); H. Kreutzmann et al., Appl. Phys. B 76, 165 (2003).

[18] S. Dettmer et al., Phys. Rev. Lett. 87, 160406 (2001).

[19] N.W. Ashcroft and N.D. Mermin Solid State Physics (Saunders College, Philadelphia, 1976).

[20] S. Stringari, Phys. Rev. A 58, 2385 (1998).

[21] W. Ketterle and N. J. van Druten, Phys. Rev. A 54, 656 (1996).

[22] H. Pu et al., Phys. Rev. A 67, 043605 (2003).

[23] Y. S. Kivshar and D. K. Campbell, Phys. Rev. E 48, 3077 (1993) and references therein. 